\title{
Theoretical study of polarization attraction in high-birefringence and spun fibers
}

\author{
Victor V. Kozlov ${ }^{1,2, *}$ and Stefan Wabnitz ${ }^{1}$ \\ ${ }^{1}$ Dipartimento di Ingegneria dell'Informazione, Universitá di Brescia, via Branze 38, 25123 Brescia, Italy \\ ${ }^{2}$ St. Petersburg University, Department of Physics, Petrodvoretz, St. Petersburg 198504, Russia \\ ${ }^{*}$ Corresponding author: victor.kozlov@email.com
}

Received September 9, 2010; revised October 13, 2010; accepted October 18, 2010; posted October 26, 2010 (Doc. ID 134891); published November 23, 2010

\begin{abstract}
Lossless polarizers are conservative nonlinear optical devices that transform unpolarized light into highly polarized light without polarization-dependent losses. The device proposed here consists of an up to 100-m-long segment of nonlinear highly birefringent or unidirectionally spun fiber pumped from the output end by an intense backwardpropagating beam. An initially unpolarized (scrambled) signal beam acquires a degree of polarization close to $100 \%$ toward the fiber output. (C) 2010 Optical Society of America
\end{abstract}

OCIS codes: $230.5440,060.4370,230.1150,230.4320$.

To the best of our knowledge, the first lossless polarizer was proposed and experimentally demonstrated in [1], where the effect of two-wave mixing in a photorefractive material was used for the amplification of one polarization component of a light beam by using the orthogonal component as a pump beam. However, the use of such a device in telecommunication applications is limited by the intrinsically slow (of the order of seconds and minutes) response time inherent to photorefractive crystals. In this respect, it appears that lossless polarizers, based on the Kerr nonlinearity of optical fibers, are more promising for fast polarization control. Indeed, fiber optics lossless polarizers were recently experimentally demonstrated. In [2], such a device, based on a 20-km-long low polarization-mode dispersion telecom (randomly birefringent) fiber counterpumped with a $600 \mathrm{~mW} \mathrm{cw}$ beam, was able to smooth at its output polarization short bursts of the input $300 \mathrm{~mW}$ signal beam. A one-beam Kerr-based lossless polarizer is reported in [3].

In this Letter, we propose and numerically validate a new configuration of lossless polarizer, which is based on a short segment of highly nonlinear and high-birefringent (HiBi) or unidirectionally spun fiber pumped from its output end by an intense cw backward-propagating beam. Our simulations reported in [4] show that, for comparable characteristics of the telecom-fiber-based setup on the one hand and the present HiBi or spun-fiber-based setup on the other hand, HiBi and spun fibers may provide a higher degree of repolarization.

The basic elements for the theoretical description of the propagation of two beams that nonlinearly interact via the polarization-dependent Kerr effect in spun silica fibers (of which the linear HiBi fiber is simply the limiting case for vanishing spin rate) can be found in [5]. In the reference frame rotating with the speed equal to the spinning rate $\tau$, the birefringence tensor $\Delta B$ of the spun fiber can be written in terms of Pauli matrices $\sigma_{1}, \sigma_{2}$, and $\sigma_{3}$, as $\Delta B(\omega)=\Delta \beta(\omega) \cos \phi\left(\cos \theta \sigma_{3}+\sin \theta \sigma_{1}+\tan \phi \sigma_{2}\right)$. Here $\Delta \beta$ is the magnitude of the intrinsic birefringence of the fiber, $\theta$ is the orientation of the birefringence axes, and $\phi=\mathrm{a} \tan (\tau / \Delta \beta)$ is the ellipticity. In a new rotating reference frame where the two polarization components of the signal beam as well as the two polarization components of the pump beam are linearly decoupled, many terms vanish as they are rapidly rotating [with a speed that is proportional to the inverse of the sum of beat lengths $L_{B}\left(\omega_{s}\right)$ and $L_{B}\left(\omega_{p}\right)$ ] on the scale of the relatively slow motion over the nonlinear length $L_{\mathrm{NL}}$. Here, $\omega_{s}\left(\omega_{p}\right)$ is the central frequency of the signal (pump) beam. Nevertheless, a few terms rotate with a speed defined by the length $L_{B}^{\prime}=\left[L_{B}^{-1}\left(\omega_{s}\right)-L_{B}^{-1}\left(\omega_{p}\right)\right]^{-1}$. Alongside their rapidly varying counterparts, such terms are responsible for four-wave mixing and are of paramount importance for achieving an efficient polarization attraction effect, which is the physical principle for the operation of lossless polarizers. The necessity to keep these terms implies that $L_{B}^{\prime} \gg L$ (where $L$ is the total fiber length), which limits us to work with beams of nearby frequencies and/or with long beat lengths, as exemplified at the end of this Letter.

The final equations of motion are conveniently formulated in terms of the three components $S_{1}^{ \pm}=\phi_{1}^{ \pm *} \phi_{2}^{ \pm}+$ $\phi_{1}^{ \pm} \phi_{2}^{ \pm *}, S_{2}^{ \pm}=i\left(\phi_{2}^{ \pm *} \phi_{2}^{ \pm}-\phi_{1}^{ \pm} \phi_{2}^{ \pm *}\right)$, and $S_{3}^{ \pm}=\left|\phi_{1}^{ \pm}\right|^{2}-\left|\phi_{2}^{ \pm}\right|^{2}$ of the signal $\mathbf{S}^{+}=\left(S_{1}^{+}, S_{2}^{+}, S_{3}^{+}\right)$and the pump $\mathbf{S}^{-}=$ $\left(S_{1}^{-}, S_{2}^{-}, S_{3}^{-}\right)$Stokes vectors. Here $\phi_{1,2}^{ \pm}$are the polarization components of the signal and pump fields in the chosen reference frame. In the limit $L / L_{B}^{\prime} \rightarrow 0$, the resulting equations read as

$$
\begin{gathered}
\partial_{\xi} \mathbf{S}^{+}=\mathbf{S}^{+} \times J_{s} \mathbf{S}^{+}+\mathbf{S}^{+} \times J_{x} \mathbf{S}^{-}, \\
\partial_{\eta} \mathbf{S}^{-}=\mathbf{S}^{-} \times J_{s} \mathbf{S}^{-}+\mathbf{S}^{-} \times J_{x} \mathbf{S}^{+} .
\end{gathered}
$$

$\xi=(v t+z) / 2$ and $\eta=(v t-z) / 2$ are propagation coordinates, where $v$ is the group speed of light in the fiber. The symbol " $x$ " denotes the vector product. Self-polarization modulation and cross-polarization modulation (XPM) tensors are both diagonal and have the form $J_{s}=\frac{1}{3} \gamma_{s s} \operatorname{diag}\left(0,0,2 \sin ^{2} \phi-\cos ^{2} \phi\right)$ and $J_{x}=\frac{2}{3} \cos ^{2} \phi \gamma_{p s}$ $\times \operatorname{diag}(1,-1,-2)$, where $\gamma_{s s} \approx \gamma_{p s} \approx \gamma$ (as the two beams have similar frequencies) with $\gamma$ as the nonlinear coefficient.

We numerically solved Eqs. (1) and (2) by the method developed in [6]. We distinguish three representative cases: (1) the case of no rotation $(\phi=0)$, which 
corresponds to the case of linearly birefringent fibers; (2) the case $\phi=\pi / 4$, which implies equality of the beat length and the spinning period; and (3) the case of rapidly spun fibers with $\phi \rightarrow \pi / 2$. The first two cases demonstrate very similar performance as demonstrated below, while the last case does not deserve further attention, because it turns out to be not favorable for the effect of interest. The reason is that the XPM tensor $J_{x}$, which is responsible for the polarization mixing and which thereby determines the efficiency of the polarization attraction, vanishes as $\phi \rightarrow \pi / 2$.

The pump power $S_{0}^{-} \equiv\left(S_{1}^{-2}+S_{2}^{-2}+S_{3}^{-2}\right)^{1 / 2}$ as well as the signal power $S_{0}^{+}$are conserved quantities. The pump power was varied between $S_{0}^{+}$and $5.5 S_{0}^{+}$. Six most representative states of polarization (SOPs) of the pump were chosen, located in close vicinity of the six poles of the Poincaré sphere: $( \pm 1,0,0),(0, \pm 1,0)$, and $(0,0, \pm 1)$. By defining the nonlinear length as $L_{\mathrm{NL}}=\left(\gamma S_{0}^{+}\right)^{-1}$, we chose $L=10 L_{\mathrm{NL}}$. The unpolarized input signal beam was modeled by a set of 110 SOPs uniformly distributed over the Poincaré sphere. For each input SOP we observed the output signal SOP a long time after launching the beams into the medium (namely after time interval $T=10000 L_{\mathrm{NL}} / v$ ) and then performed the average of the quantities of interest over all output signal SOPs. Thus, we are actually dealing with scrambled beams.

In monitoring the performance of the polarizer, two quantities are of interest: (1) the signal SOP as a function of the pump SOP and the pump power and (2) the degree
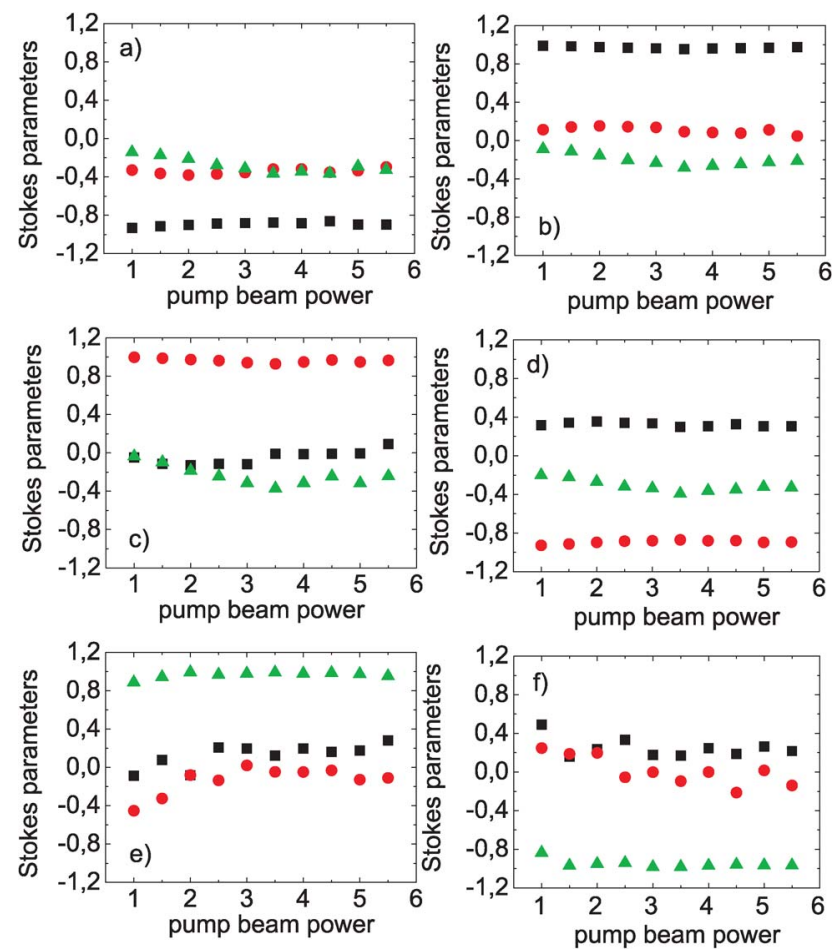

Fig. 1. (Color online) Components of the (normalized) Stokes vector of the output signal beam as a function of the pump beam power, $S_{1}^{+}$(black squares), $S_{2}^{+}$(red circles), $S_{3}^{+}$(green triangles), for the six input SOPs of the pump: (a) (-0.99, 0.1, $0.1)$, (b) $(0.99,0.1,0.1)$, (c) $(0.1,-0.99,0.1)$, (d) $(0.1,0.99$, $0.1)$, (e) $(0.1,0.1,-0.99)$, (f) $(0.1,0.1,0.99) . \phi=0$. The case with $\phi=\pi / 4$ (not shown) demonstrates very similar qualitative as well as quantitative results. of polarization (DOP) of the outcoming signal beam as a function of pump power. Clearly, the lossless polarizer performs efficiently when the output DOP is close to unity.

Figure 1 demonstrates that the signal SOP depends on the pump $\overline{\mathrm{SOP}}$, but it is virtually independent of the pump power in the range $[1,5.5] S_{0}^{+}$. The dependence on the pump SOP can be used for polarization control, while the tolerance to pump intensity fluctuations is a favorable feature for applications. In general, we found that the signal SOP is not exactly attracted to the pump SOP, as pointed out also for isotropic fibers (see [7]).

Figures 2 and 3 show the DOP of the output signal (at $z=L)$ as a function of the pump power for the same conditions as used for the generation of the plots of Fig. 1 . The polarizer performance is close to ideal $(D=0.99$ in the best case) for the case when the pump SOP is $(0.99$, $0.1,0.1)$. Naturally, the DOP quickly degrades when $S_{0}^{-}$ drops below $S_{0}^{+}$(not shown). Good performance is also observed for the pump SOPs $(-0.99,0.1,0.1)$ and $(0.1$, $\pm 0.99,0.1)$ in the range of pump powers $S_{0}^{-}=[2,5] S_{0}^{+}$. However, the polarizer performs very poorly when the pump SOP contains an appreciable $S_{3}$ component. We have also observed that the repolarization of the signal beam toward the output at $z=L$ is accompanied with simultaneous scrambling of the counterpropagating pump beam toward $z=0$. The effect of depolarization of the pump beam was previously reported in $[\underline{8}, \underline{9}]$ for the case of isotropic fibers.

Based on all these considerations and on extensive additional numerical simulations not demonstrated here, we may conclude that strong polarization attraction takes place whenever the pump beam does not contain any substantial share of the $S_{3}$ component. Physically, this means that the pump power should be equally split between the two elliptical polarization eigenmodes (as far as the linear response is concerned) of the unidirectionally spun fiber. Indeed, the definition of the pump SOP is related to the reference frame $\left(e_{1}, e_{2}\right)$, in which the linear part of the equations of motion for the polarization components of the pump and the signal beams are decoupled. In its turn, such a frame is related to the rotating laboratory reference frame $\left(e_{x}, e_{y}\right)$ by the transformation

$$
\left(\begin{array}{l}
e_{1} \\
e_{2}
\end{array}\right)=\left(\begin{array}{cc}
\cos \frac{\phi}{2} & i \sin \frac{\phi}{2} \\
i \sin \frac{\phi}{2} & \cos \frac{\phi}{2}
\end{array}\right)\left(\begin{array}{l}
e_{x} \\
e_{y}
\end{array}\right) .
$$
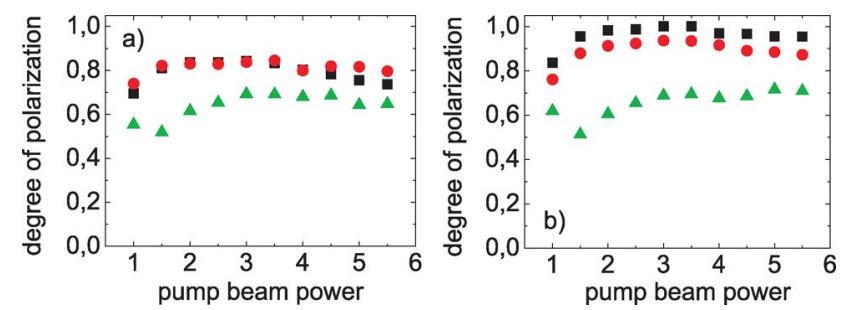

Fig. 2. (Color online) DOP $D$ of the output signal beam as a function of the pump beam power for six input SOPs of the pump (same as in Fig. 1): (a) $(-0.99,0.1,0.1)$ (black squares), $(0.1,-0.99,0.1)$ (red circles), (0.1, 0.1, -0.99) (green triangles); (b) $(0.99,0.1,0.1)$ (black squares), $(0.1,0.99,0.1)$ (red circles), $(0.1,0.1,0.99)$ (green triangles). $\phi=0$. 

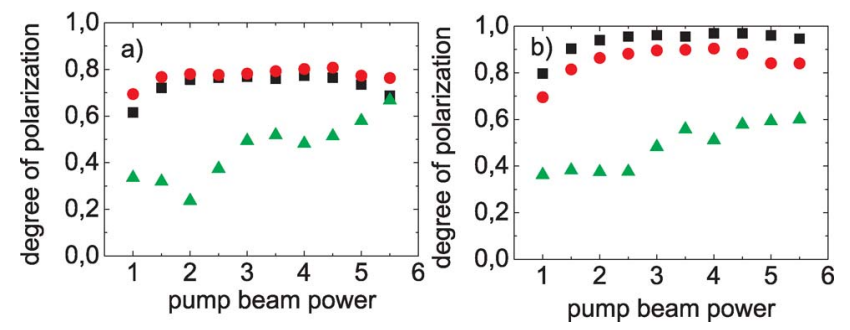

Fig. 3. (Color online) Same as in Fig. 2 for $\phi=\pi / 4$.

Given that $S_{3}=0$ implies equal shares of the $e_{1}$ and $e_{2}$ components, one may restore the pump SOP in the laboratory frame by using the transformation of Eq. (3).

Coming back to the main requirement $L_{B}^{\prime} \gg L$, we can rewrite it as a condition for the difference of pump and signal wavelengths $\lambda_{s}-\lambda_{p} \ll 2 \pi \lambda_{p} /\left[\Delta \beta\left(\lambda_{p}\right) L\right]$, where we assumed that $\Delta \beta \propto \lambda^{-1}$. For $L=100 \mathrm{~m}, \lambda_{p}=1.55 \mu \mathrm{m}$, and $\Delta \beta\left(\lambda_{p}\right)=1 \mathrm{~cm}^{-1}$, we get the estimate $\lambda_{s}-$ $\lambda_{p} \ll 1 \mathrm{~nm}$.

Note that, in our modeling, we considered spun fibers with a birefringence value that is constant along the entire fiber length. Such a model works well for distances not exceeding the birefringence correlation length, which measures the length scale of the fiber birefringence stochasticity. This length rarely exceeds $100 \mathrm{~m}$. To get substantial nonlinear mixing of polarizations inside a fiber of only 100-m-long for average signal powers lower than $1 \mathrm{~W}$, one is forced to use highly nonlinear fibers. This can be nonlinear photonic crystal fiber with $\gamma=0.1(\mathrm{~W} \cdot \mathrm{m})^{-1}$ as used in [10], tellurite photonic crystal fiber with $\gamma=5.7(\mathrm{~W} \cdot \mathrm{m})^{-1}$ as used in [11], or chalcogenide fibers.
In conclusion, we demonstrated the presence of the polarization attraction effect in a short segment of a highly nonlinear $\mathrm{HiBi}$ and/or spun fiber and identified the pump SOPs for which this attraction is most efficient.

We thank J. Fatome for sending us a preprint of [2] prior to its publication. This work was carried out in the framework of the "Scientific Research Project of Relevant National Interest" (PRIN 2008) entitled "Nonlinear cross-polarization interactions in photonic devices and systems" (POLARIZON).

\section{References}

1. J. E. Heebner, R. S. Bennink, R. W. Boyd, and R. A. Fisher, Opt. Lett. 25, 257 (2000).

2. J. Fatome, S. Pitois, P. Morin, and G. Millot, Opt. Express 18, 15311 (2010).

3. A. Picozzi, Opt. Express 16, 17171 (2008).

4. V. V. Kozlov, J. Nun̄o, and S. Wabnitz, "Theory of lossless polarization attraction in telecommunication fibers," J. Opt. Soc. Am. B (to be published).

5. S. Wabnitz, IEEE Photonics Technol. Lett. 21, 875 (2009).

6. C. Martijn de Sterke, K. R. Jackson, and B. D. Robert, J. Opt. Soc. Am. B 8, 403 (1991).

7. E. Assémat, S. Lagrange, A. Picozzi, H. R. Jauslin, and D. Sugny, Opt. Lett. 35, 2025 (2010).

8. S. Pitois, A. Picozzi, G. Millot, H. R. Jauslin, and M. Haelterman, Europhys. Lett. 70, 88 (2005).

9. S. Pitois, J. Fatome, and G. Millot, Opt. Express 16, 6646 (2008).

10. A. A. Amorim, M. V. Tognetti, P. Oliveira, J. L. Silva, L. M. Bernardo, F. X. Kärtner, and H. M. Crespo, Opt. Lett. 34, 3851 (2009).

11. M. Liao, C. Chaudhari, G. Qin, X. Yan, T. Suzuki, and Y. Ohishi, Opt. Express 17, 12174 (2009). 